\title{
Emergent dynamics of cardiomyocyte clusters on deformable polymeric substrates
}

\author{
Sandeep V. Anand ${ }^{1}$ and Taher A. Saif ${ }^{1,}$ a) \\ Department of Mechanical Science and Engineering, University of Illinois at Urbana-Champaign, Urbana, \\ Illinois 61801, USA
}

(Dated: 15 June 2015)

\begin{abstract}
Contractile dynamics of primary cardiomyocyte clusters is studied by culturing them on deformable thin polymeric films. The cell clusters beat and generate sufficient forces to deform the substrates out of plane. Over time, the clusters reorient their force dipoles along the direction of maximum compliance. This suggests that the cells are capable of sensing substrate deformations through a mechanosensitive feedback mechanism and dynamically reorganizing themselves.
\end{abstract}

Keywords: Cardiomyocyte, force dipole, thin films, mechanosensitive, dynamics, compliance

In recent years, cardiomyocytes have emerged as a popular choice for bioactuators for powering biological machines consisting of soft polymeric scaffolds at the micro and macro scales ${ }^{1-7}$. This is owing to their unique ability to generate spontaneous, synchronous contractions consuming only glucose as their primary energy source ${ }^{8}$. Most of the biological machine designs reported in the literature use cardiomyocytes conjugated with biocompatible soft polymers like polydimethylsiloxane (PDMS) and hydrogels to produce some form of locomotion by converting chemical energy of the cardiomyocytes to mechanical energy. The mode of locomotion may vary, but the fundamental mechanism that these biological machines exploit to achieve locomotion stems from cell substrate interactions leading to large deformations of the substrates (relative to the cell size). However, the effect of such large scale, dynamic deformation of the substrates on the cellular and cluster level organization of the cardiomyocytes remains elusive.

It is known that cardiomyocytes are mechanosensitive to their local environment and are capable of sensing the substrate elasticity, among other mechanical factors, to remodel and reorganize their internal structures. For example, cardiomyocytes generate highest force when cultured on hydrogel substrates mimicking the elasticity $(\sim 10 \mathrm{kPa})$ of the heart tissue. The sarcomere alignment and beating frequency of cardiomyocytes are also influenced by substrate stiffness ${ }^{9-12}$. When cultured on polymeric micropillar substrates, the cells generated higher contraction forces on stiffer pillars at the expense of contraction rates. The sarcomere lengths and z-band widths also increase with stiffness ${ }^{13}$.

Mechanical microenvironment plays an important role in the modulation of the adhesion structures of cardimyocytes with substrates. The size and frequency of adhesion sites increase in response to externally applied stretch and cell contractility ${ }^{14-16}$. Force is

a)Electronic mail: saif@illinois.edu; Tel: 217-333-8552 estimated to be about $15 \mathrm{nN}$ per focal adhesion site, and the net force dipole per cell is about 200-500 $\mathrm{nN}^{17,18}$. When cultivated on soft, flexible silicone membranes, the cells generate pleat like wrinkles with a spacing of $1.9 \mu \mathrm{m}$. The observed pleats align with the z-disks of the cells ${ }^{19}$.

The aforementioned observations of cardiomyocyte mechanosensitivity to their local microenvironment raises the possibility that the large dynamic deformations of the biological machines may induce cellular reorganization in the contraction dynamics of the cardiomyocyte clusters. The objective of this study is to explore this possibility by plating clusters of cardiomyocytes on deformable film substrates with anisotropic compliance.

One-dimensional polydimethylsiloxane (PDMS) cantilever beams (henceforth referred to as filaments) were developed using a technique developed earlier ${ }^{1,2,20}$. The filaments were incubated in fibronectin (BD Bioscience, $25 \mu \mathrm{g} / \mathrm{mL}$ ) extracellular matrix (ECM) protein for 3 hours and rinsed in PBS three times. The ECM functionalized filaments were transferred to a custom prepared substrate which was previously incubated in Pluronic F127 (1\% in PBS, Sigma-Aldrich) for 20 mins to prevent cell attachment. The substrate consisted of two square silicon chips, $500 \mu \mathrm{m}$ thick, and $4 \mathrm{mmX} 4 \mathrm{~mm}$ in size. They were glued to a polystyrene petri-dish, and were separated by a distance equal to the length of the filaments. Each filament was placed manually on two chips such that they form a straight bridge between them. The ends of the filaments attached to the silicon chips through stiction, (Fig. 1a-d).

Primary cardiomyocytes extracted from neonatal rats were plated on the filaments. Several cardiomyocytes congregated and formed clusters on different locations of the filaments within $12 \mathrm{hrs}$ of cell plating. The cluster sizes typically varied between 70 and $80 \mu \mathrm{m}$ in diameter. A schematic of the filament configuration and cell plating methodology is shown in Figs. 1(a-c). Filaments of two 

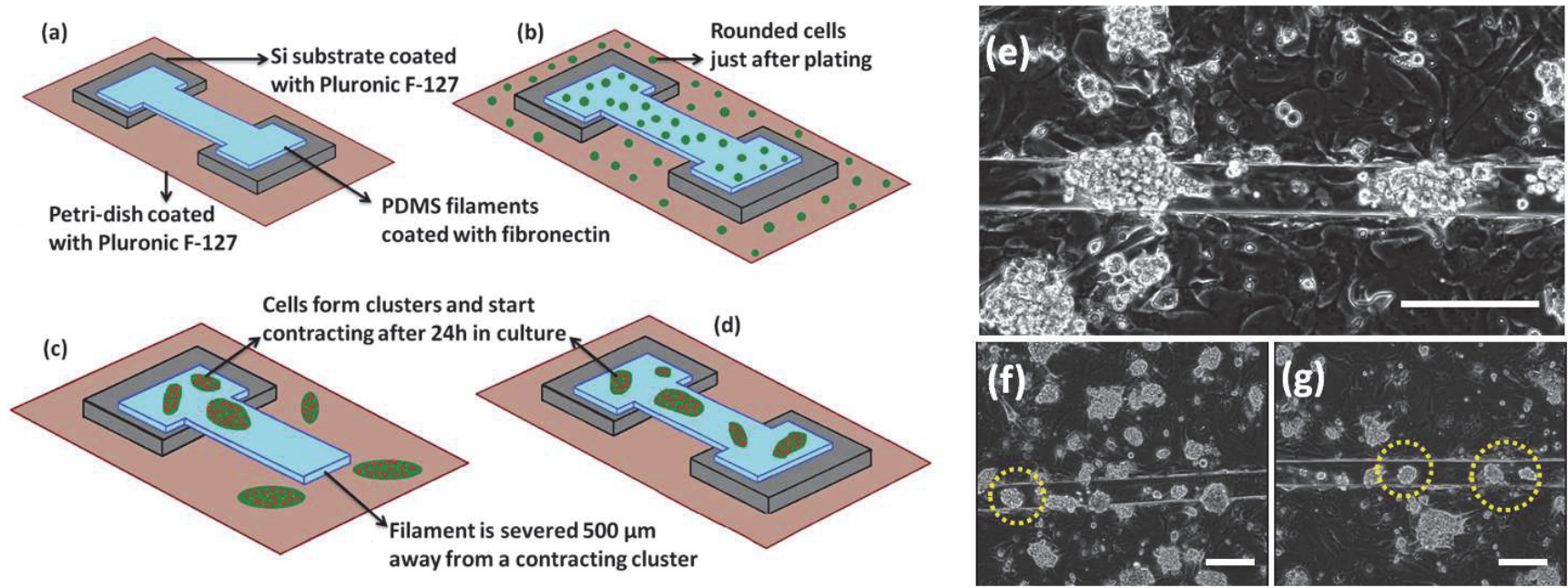

FIG. 1. Experimental platform to study cell-substrate dynamic feedback. (a) Initial configuration of the fibronectin functionalized filament on the substrate before cell plating. (b) Primary cardiomyocyte cells from neonatal rats are plated on the filaments. (c) The filament is severed $\mu \mathrm{m}$ away from a contracting cardiomyocyte cluster at the base of the cantilever after 24hrs of cell plating. (d) Filament fixed at both ends (e) Cardiomyocyte clusters on a narrow filament (30 $\mu \mathrm{m}$ wide). (f-g) Cell clusters on wide filaments $(90 \mu \mathrm{m}$ wide). Cell clusters are well within the width of the filament. (Scale bar: $100 \mu \mathrm{m})$

different widths were chosen for the study. The narrow filaments were between $30-40 \mu \mathrm{m}$ in width and 7-10 $\mu \mathrm{m}$ in thickness, while the wide filaments were between 90-100 $\mu \mathrm{m}$ in width and $10-15 \mu \mathrm{m}$ in thickness. The wider filaments contain the clusters completely within their boundaries so that their dynamics is not affected by the edges (Figs. 1(f-g)), while the cluster orientation is affected by the geometry of the narrow filaments (Fig. $1(\mathrm{e})$ ).

In this study, only the filaments with cell clusters near $(50 \mu \mathrm{m})$ the anchored ends of the filaments were chosen. Within this group, two types of filaments were studied. (1) Cantilevered filaments: these filaments were severed $500 \mu \mathrm{m}$ away from the beating cluster so that they become cantilevers fixed at one end (anchor) with a beating cell cluster near the anchor where the in-plane compliance is anisotropic (see supplementary videos S3 and S4 for narrow and wide filaments respectively). The compliance is low along the transverse direction due to the restraining effect of the anchor, but is high along the longitudinal direction. (2) Fixed-fixed filament: these filaments were not severed, but instead were left intact (see supplementary videos S1 and S2 for narrow and wide filaments respectively). Beating clusters on both the cantilevered and fixed-fixed filaments were video recorded for $1 \mathrm{~min}, 24$ (day 1 ) and $48 \mathrm{hrs}$ (day 2) after severing the filaments ( $48 \mathrm{hrs}$ and $72 \mathrm{hrs}$ after cell plating respectively) using phase contrast microscopy.

Cardiomyocyte clusters beat as a single actuator through synchronous beating of individual cells within the cluster. By simply observing the dynamics of the cluster one can identify its dominant contraction
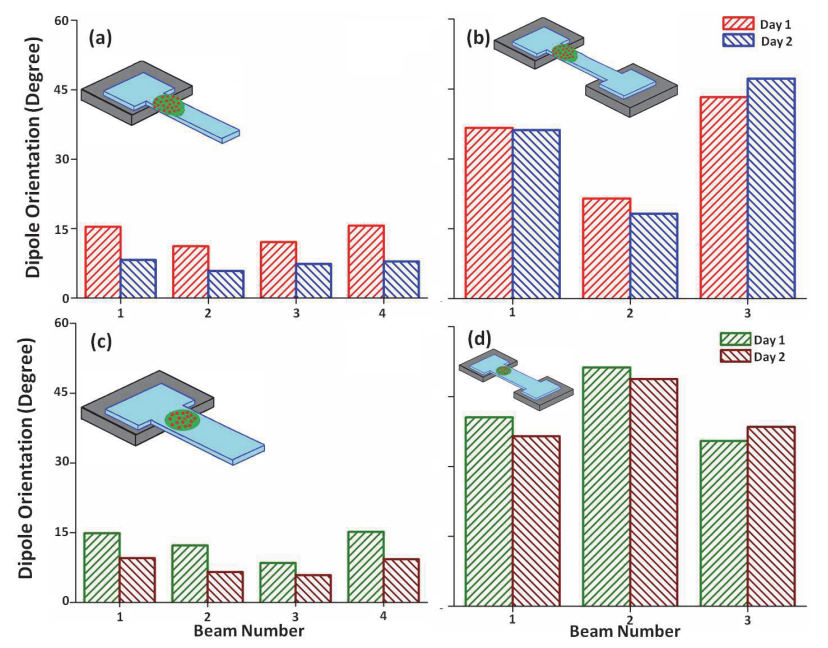

FIG. 2. Directions of contractile dipoles of cell clusters on filaments (see Fig. 1) become biased within $48 \mathrm{hrs}$. (a) Contractile force dipole directions of clusters on four different narrow cantilever filaments (free to deform) after 1 and 2 days of release (see Fig. 1). (b) Dipole directions on three different narrow fixed-fixed filaments. (c) Dipole directions on wide cantilever filaments, and (d) wide fixed-fixed filaments.

direction, i.e., the direction of its dominant force dipole. Here, the direction was determined from a line through two points within the cluster with highest opposing displacements. The angle between this line and the longitudinal direction of the filament gives the dipole direction. Thus, smaller angles (closer to $0^{\circ}$ ) mean that the cluster is contracting along the length of the filaments, and larger angles (closer to $90^{\circ}$ ) mean that they are contracting transversely. 


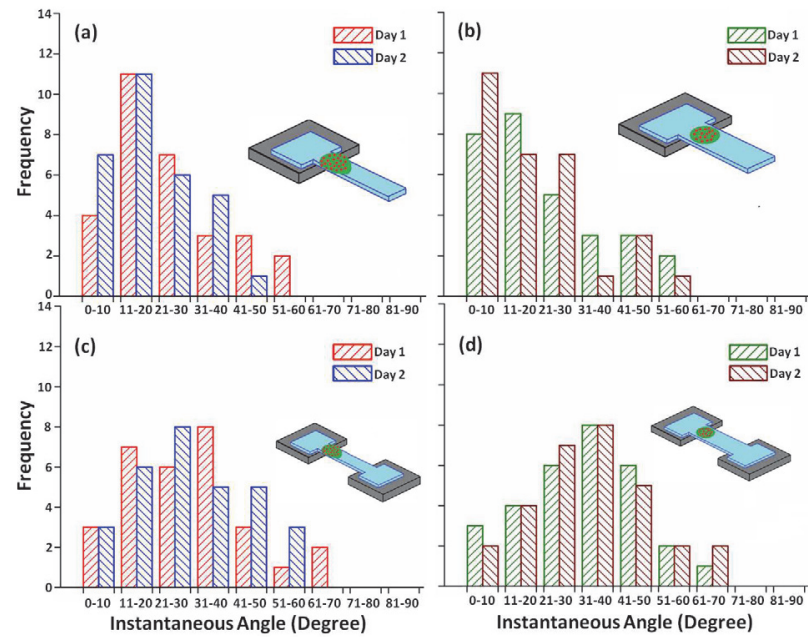

FIG. 3. Direction of motion of individual points tracked on cardiomyocyte clusters on (a) narrow cantilever filament (b) wide cantilever filament (c) narrow fixed filament and (d) wide fixed filament.

Fig. 2 shows dipole orientation angles for four cell clusters on 4 cantilever filaments, and three clusters on fixed-fixed filaments. The dipole angles are much smaller (within $15^{\circ}$ ) for free cantilevers, both narrow and wide, compared to those $\left(20^{\circ}-40^{\circ}\right)$ for the fixed-fixed cases. This indicated that the cell clusters were mechanosensitive to substrate compliance and suitably reoriented their dipoles based on the substrate deformation feedback. Furthermore, the dipole angles of clusters on the cantilever filaments reduced over time from about $15^{\circ}$ to less than $10^{\circ}$ between day 1 and 2 (48 and $72 \mathrm{hrs}$ of cell plating) implying a continuous biased reorganization process. Note that the cantilevers were made by severing the fixed-fixed filaments after $24 \mathrm{hrs}$ of cell plating. For the fixed-fixed case where the filaments were left intact after cell plating, dipole directions did not change with time. The fact that cell clusters on both the narrow and wide filaments showed similar reorganization of dipole directions with time implies that substrate compliance, and not geometry, was the dominant cue for cell response.

In order to explore the contributions of many cells to the dynamics of the cluster, we traced the motion of multiple points on the clusters. Fig. 3 shows the distribution of angles made by the points (30 per cluster) with respect to the longitudinal axis of the filaments. Figs. 3(a-b) are histograms for two different clusters on a narrow and a wide cantilever filament respectively while Figs. 3(c-d) represent histograms for two different clusters on narrow and wide filaments, fixed at both ends. As can be seen, most of the points on the free cantilever filaments (in both narrow and wide filaments) had angles within $0-50^{\circ}$ while those on the fixed-fixed filaments had angles within $10-70^{\circ}$, with mean angles of $18^{\circ}$ and $42^{\circ}$ respectively, consistent with the dominant
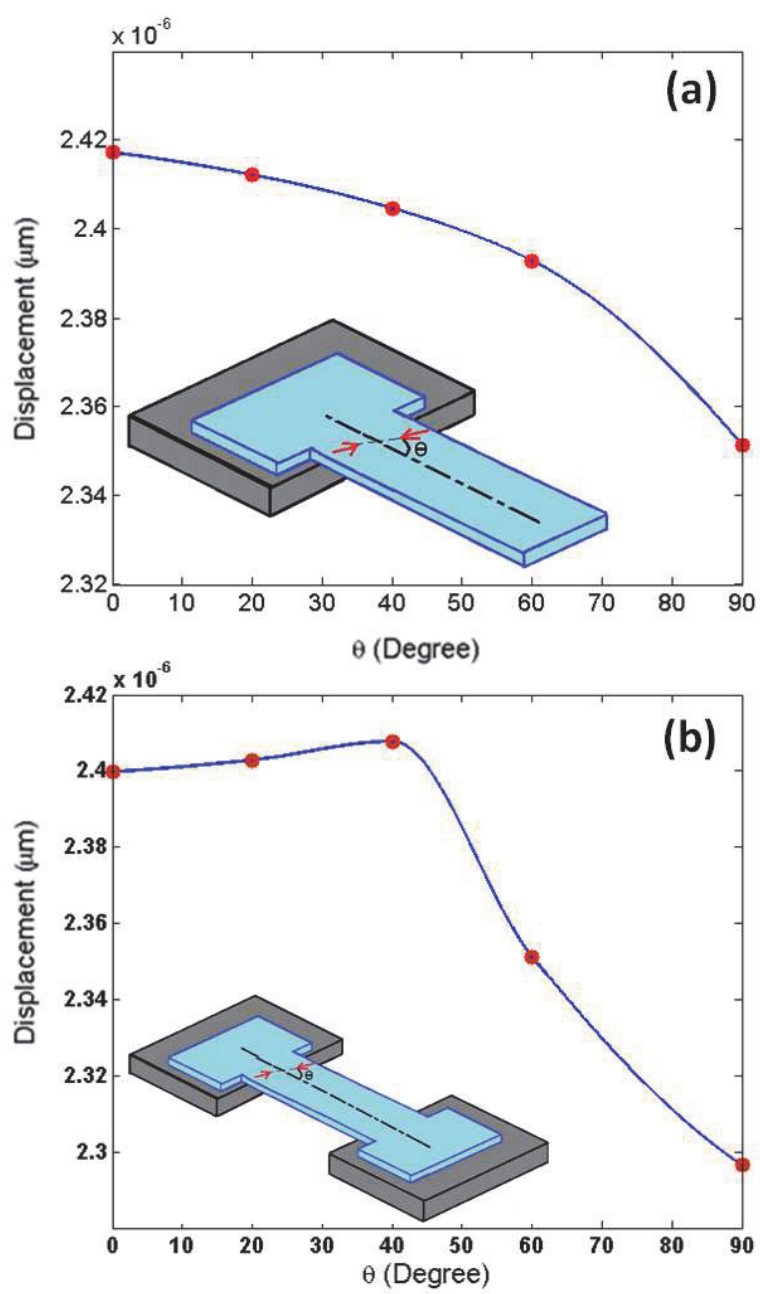

FIG. 4. In-plane compliance of the cell substrate filaments near the support along different directions evaluated by finite elements analyses of fixed-fixed and cantilever filaments. Compliance is measured by the relative displacements of two points subjected to unit forces $(1 \mu \mathrm{N})$ forming a dipole. The forces are applied along the plane of the substrate, mimicking the dipole forces of cell clusters. Compliance is highest along the longitudinal direction for the cantilever filament, but it decays with increasing angle. For the fixed-fixed case, compliance is highest along $40^{\circ}$. It decays slowly with decreasing angle, but rapidly with increasing angle. Cell clusters align their dominant dipoles (Fig. 2) along the compliant directions of the filaments.

dipole directions of Fig 2. This indicates that the cells within the clusters on the free cantilever filaments reoriented themselves along the longitudinal axis of the filaments as a result of the feedback from substrate deformations.

In the above study, it was hypothesized that the cell clusters on deformable filaments would beat along the most compliant direction to maximize the contractile displacements. For cantilever filaments, this meant the 
longitudinal direction $\left(0^{\circ}\right)$. It is found that the clusters on cantilevers indeed beat along directions close to the longitudinal direction. With time, they align more with the longitudinal direction. However, the angles of cardiomyocyte clusters on the fixed-fixed filaments were curiously biased towards $20-40^{\circ}$. This observation led us to speculate that the preferred orientation might originate from the asymmetric compliance of the fixed-fixed filament. In search of this compliance, a finite element methods (FEM) analysis of both the fixed-fixed and fixed-free filaments was performed using a commercially available FEM software (ANSYS). Dipoles with unit forces separated by $45 \mu \mathrm{m}$ were applied on the surface of the filament near the anchor to mimic the contractile cell cluster. Analysis was performed for various dipole orientations within $0-90^{\circ}$ for both the free cantilever and the fixed-fixed filaments. For a given dipole orientation, the relative displacement between the dipole force points gives a measure of compliance for that orientation. In case of the free cantilever, compliance is highest along the longitudinal direction (Fig. 4a), as expected. For the fixed-fixed case, however, compliance is highest along $40^{\circ}$ (Fig. 4b), although compliance decreases slightly with decrease of dipole angle from $40^{\circ}$. Compliance decreases rapidly after $40^{\circ}$, and is lowest along $90^{\circ}$ due to the constraint of the fixed anchors. This offers a possible explanation of the emerged, dipole orientation along $20-40^{\circ}$ for the fixed-fixed case, and smaller angles for the cantilever case. In addition, the fixed-fixed filaments were intact since the cells were plated. Hence no change in dipole orientation was observed between day 2 and 3 after plating. The fixed-free cantilever was obtained by severing the fixed-fixed cantilever 1 day after cell plating. Hence, the cell clusters re-oriented their dipole directions after the release of the cantilevers over the following two days (day 2 and 3 after plating), i.e., the cell clusters sense the most compliant direction for both the free and the fixed filaments and their dominant dipole direction emerges along the compliant direction (see supplementary figures S1 and S2).

In conclusion, the dynamic cross talk between contractile neonatal rat cardiomycyte cluster and a deformable substrate with anisotropic compliance is explored. The cell clusters are found to be sensitive to the anisotropy. The clusters gradually orient their contractile dipole direction along the most deformable direction of the substrate. The emergence of this orientation occurs over many cycles of contractile beating. This implies a dynamic feedback mechanism through which the cells sense the substrate deformations they produce by beating, and in turn optimize their contraction. The most deformable direction appears to be the most optimum for the substrates studied in this paper.

This project was funded by the National Science Foundation (NSF), Science and Technology Center on Emergent Behaviors in Integrated Cellular Systems (EBICS) Grant CBET-0939511.

${ }^{1}$ B. J. Williams, S. V. Anand, J. Rajagopalan, and M. T. A. Saif, Nature communications 5, 3081 (2014).

${ }^{2}$ S. V. Anand, M. Y. Ali, and M. T. A. Saif, Lab Chip 15, 1879 (2015).

${ }^{3}$ V. Chan, K. Park, M. B. Collens, H. Kong, T. A. Saif, and R. Bashir, Scientific reports 2, 857 (2012).

${ }^{4}$ V. Chan, H. H. Asada, and R. Bashir, Lab on a chip 14, 653 (2014).

${ }^{5}$ C. Cvetkovic, R. Raman, V. Chan, B. J. Williams, M. Tolish, P. Bajaj, M. S. Sakar, H. H. Asada, M. T. A. Saif, and R. Bashir, Proceedings of the National Academy of Sciences of the United States of America 111, 10125 (2014).

${ }^{6}$ R. D. Kamm and R. Bashir, Annals of biomedical engineering 42, 445 (2014).

${ }^{7}$ J. C. Nawroth, H. Lee, A. W. Feinberg, C. M. Ripplinger, M. L. McCain, A. Grosberg, J. O. Dabiri, and K. K. Parker, Nature biotechnology 30, 792 (2012).

${ }^{8}$ Y. Tanaka, Y. Tanaka, K. Sato, K. Sato, T. Shimizu, T. Shimizu, M. Yamato, M. Yamato, T. Okano, T. Okano, T. Kitamori, and T. Kitamori, Biosensors \& bioelectronics 23, 449 (2007).

${ }^{9}$ J. G. Jacot, A. D. McCulloch, and J. H. Omens, Biophysical journal 95, 3479 (2008).

${ }^{10}$ J. G. Jacot, J. C. Martin, and D. L. Hunt, Journal of Biomechanics 43, 93 (2010).

${ }^{11}$ P. Bajaj, X. Tang, T. A. Saif, and R. Bashir, Journal of Biomedical Materials Research - Part A 95, 1261 (2010).

${ }^{12}$ A. J. Engler, C. Carag-Krieger, C. P. Johnson, M. Raab, H.-Y. Tang, D. W. Speicher, J. W. Sanger, J. M. Sanger, and D. E. Discher, Journal of cell science 121, 3794 (2008).

${ }^{13}$ A. G. Rodriguez, S. J. Han, M. Regnier, and N. J. Sniadecki, Biophysical Journal 101, 2455 (2011).

${ }^{14}$ D. G. Simpson, M. L. Decker, W. A. Clark, and R. S. Decker, Journal of Cell Biology 123, 323 (1993).

${ }^{15}$ W. W. Sharp, D. G. Simpson, T. K. Borg, A. M. Samarel, and L. Terracio, The American journal of physiology 273, H546 (1997).

${ }^{16}$ C. E. Miller, K. J. Donlon, L. Toia, C. L. Wong, and P. R. Chess, In vitro cellular \& developmental biology. Animal 36, 633 (2000).

${ }^{17}$ N. Q. Balaban, U. S. Schwarz, D. Riveline, P. Goichberg, G. Tzur, I. Sabanay, D. Mahalu, S. Safran, A. Bershadsky, L. Addadi, and B. Geiger, Nature cell biology 3, 466 (2001).

${ }^{18}$ C. M. Cesa, N. Kirchgeß ner, D. Mayer, U. S. Schwarz, B. Hoffmann, and R. Merkel, Review of Scientific Instruments $\mathbf{7 8}$ (2007), 10.1063/1.2712870.

${ }^{19}$ B. A. Danowski, K. Imanaka-Yoshida, J. M. Sanger, and J. W. Sanger, Journal of Cell Biology 118, 1411 (1992).

${ }^{20}$ J. Rajagopalan and M. T. A. Saif, Journal of Microelectromechanical Systems 22, 992 (2013). 